\title{
LI. On the vibratory movements which magnetic and nonmagnetic bodies experience under the influence of external and transmitted electric currents
}

\section{Professor De la Rive}

To cite this article: Professor De la Rive (1849) LI. On the vibratory movements which magnetic and nonmagnetic bodies experience under the influence of external and transmitted electric currents, Philosophical Magazine Series 3, 35:238, 422-434, DOI: 10.1080/14786444908646383

To link to this article: http://dx.doi.org/10.1080/14786444908646383

册 Published online: 30 Apr 2009.

Submit your article to this journal ๘

山l Article views: 2

Q View related articles $\longleftarrow$ 
LI. On the Vibratory Movements which Magnetic and Nonmagnetic Bodies experience under the infuence of external and transmitted Electric Currents. By Professor DE LA RIVE*. I. WERTHEIM has published in the Amnales de some further researches on the sounds produced by the electric current. He directs attention to the fact that these sounds are of two kinds ; those proceeding from the action of an external current which magnetizes an iron bar or wire, and those produced by a current transmitted either through an iron bar or wire. The sounds of the first kind were discovered as early as 1837 by Mr. Page, and afterwards investigated by MM. Marian, Matteucci, Wartmann † and myself. I was the first who indicated the existence of the sounds of the second kind, which Mr. Beatson discovered nearly at the same time as myself, and which have also been the object of the researches of the physicists named above, and likewise of some others. M.Wertheim, in 1844, demonstrated that the electric current and magnetization produce a diminution of the coefficient of elasticity on the bodies which are submitted to their influence; but he thought that he perceived in the sounds produced by these two causes rather a mechanical effect than a molecular phænomenon $\ddagger$, attributing the sounds which the magnetization determines to the attractive action of the helix on the wire or on the bar of iron, and those caused by the transmitted current to a kind of shock which that transmission effected upon the conducting metal. Admitting, in the case of magnetization, in part the cause pointed out by M. Wertheim, I showed that the molecular action has its own, and that, in the case of the transmitted current, it alone is active. An experiment by M.Guillermin, and the researches of M.Wartmann, confirmed this view, if not on all points, at least on the greatest number. Lastly, in a more recent memoir, I succeeded in showing that, under the influence of a magnet or a helix traversed by a continuous current, all conducting bodies are capable of producing a sound when they transmit a discontinuous current.

In a memoir just published, M. Wertheim, resuming the subject, establishes first, by numerous experiments made with great care, that in magnetization there is a mechanical traction due to a longitudinal and to a transversal component; that the latter becomes null when the iron bar is in the centre

* From the Annales de Chimie et de Physique for June 1849.

+ Phil. Mag. vol. xxviii. p. 544.

\$. Comptes Rendus des Séances del'Académie des Sciences, vol. xxiii. p.336. 
of the helix, but that in all cases the longitudinal one subsists; and that this force, acting in the direction of the axis, exists equally with a transmitted current. It must produce a longitudinal sound, whether it tends to lengthen or abruptly to shorten the bar; the transversal sound can only arise from the external current, and in an excentric position of the bar. Passing then to the examination of the sounds themselves, M. Wertheim finds their explanation in the mechanical actions which we have just indicated; he does not therefore think that either the magnetization or the transnission of the electric current produces vibrations of a particular kind; but he supposes that the mechanical actions which they engender determine accidentally these longitudinal or transversal vibrations, as any other cause might do. He admits, however, that there is in some cases a dull noise (bruit sec), like a kind of crepitation, which is propagated at the moment when the current traverses an iron bar or wire; and he concludes by remarking, that there are still in this class of phænomena many obscure points, especially that which relates to the manner in which a current traversing the iron produces in it a mechanical shock.

M. Wertheim's new observations, of which I have thus given a summary, have induced me to resume my experiments, and to investigate more closely the curious phænomena in question.

It appears to me evident now, that the point which I have sought to establish in my first memoir of $1845^{*}$, namely that the magnetization or the passage of the electric current produces a molecular derangement, is no longer disputed. I admit, on the other hand, that $I$ have perhaps attached too much importance to the nature of the sounds produced, and to the influence of certain causes, such as tension, which occasions them to vary. It may be, in fact, that the wire, once set in vibration by either the external or transmitted current, a simple friction against any metallic piece next to the monochord may suffice, if not completely, at least in many cases, to account for the variety of the remarkable sounds which are heard, especially when well-annealed iron wires are used †.

My attention has therefore been especially directed, in

* Comptes Rendus des Séances de l'Académie des Sciences, vol. xx. p.1287; and Archives de l'Electricité, vol. v. p. 200.

$\uparrow$ 'The Comptes Rendus de l'Académie des Sciences, vol. xxvii. p. 457, contains the extract of a memoir by $M$. Duhamel on the multiple sounds of bodies, which it appears to me may be well applied to the study of the sounds produced in the iron wires by simple molecular movements. See Phil. Mag,, vol, xxxiv, p. 415. 
the researches which $I$ have just made, to the investigation of the cause of the fundamental fact, that is to say, of the oscillations which the particles of bodies experience around their position of equilibrium by the action of either external or transmitted currents. With this view, I first submitted to experiment bodies, like iron, susceptible of magnetism, and then other conducting bodies which are not magnetic.

\section{$\S$ 1. Examination of the Vibrations produced in magnetic bodies by either external or transmitted currents.}

On placing very fine iron-filings in the interior of a helix with the axis vertical, these filings are seen to form themselves, under the influence of the current traversing the helix, into small pyramids, elongated in the direction of the axis, which are destroyed and re-formed rapidly when the current is intermittent. The action of the current of the helix on these filings consists, therefore, in distributing them under the form of an elongated thread parallel to the axis,-a thread which the weight alone prevents being as long as the helix itself.

This experiment, which I have already described, and which succeeds as well with very fine iron powder as with ironfilings, proves that the particles of iron under the influence of external magnetization tend to approach each other in the transversal direction, and to extend in the longitudinal direction. It is probable that this phænomenon is due to the form of the elementary particles of the iron, and to the manner in which they are polarized. One thing is certain, that it accounts for what passes in an iron bar or wire submitted to the influence of the intermittent current of the helix. The particles of the iron contending with the cohesion arrange themselves in the longitudinal direction when the current acts, and return to their primitive position as soon as it ceases; from this results a series of oscillations which are isochronous with the interruptions of the current. This manner of viewing the phænomenon entirely agrees with the contraction in the transversal direction accompanied by an extension in the longitudinal direction, which Mr. Joule observed in iron wires and bars sulumitted to magnetization*.

The same physicist has also remarked that when the wire is much stretched, the magnetization occasions a shortening instead of a lengthening, - an effect which agrees with the cessation of the sounds I have observed when the tension becomes too strong.

All these effects are much more decided in soft iron than

* Phil. Mag. Feb. 1847 and April 1847. 
in bardened iron and steel ; the cause of which is, that in soft iron the particles are much more mobile around their position of equilibrium. Soft iron and steel magnetized by the action of a magnet or a second helix, exhibit weaker vibrations when the external current tends to magnetize them in the direction in which they are already magnetized, and stronger in the contrary case. The passage of a continuous current through an iron wire modifies the sound which the iutermittent action of the external current of the helix causes it to emit, provided that the transmitted current is very strong and the iron very soft.

I shall not enlarge more on this first case, which I think is now well explained; I pass to that of the transmitted current.

An electric current transmitted intermittently through a wire of very soft iron and of small diameter (of 1 to 3 millimetres), determines in it vibrations as strong as those which are produced in it by the same current acting externally under the most favourable conditions. If the iron wire becomes larger, or if it is more hardened, or if it is a steel wire, the effect of the transmitted current is less than that of the external current*. With rods of soft iron, of 4 to 5 millimetres in diameter, the transmission of the continuous current does not completely extinguish, but only diminishes, the effect of the discontinuous current. If the rods are very large, the diminution becomes less perceptible, unless very strong batteries are employed. In the preceding experiments, I made use of two of Grove's batteries of five cells, one for the continuous current, the other for the discontinuous one.

The same experiment made on steel rods and wires gave me precisely contrary results. The sound produced by the transmission of the discontinuous current is weak; but it is increased, instead of being dininished, by the passage of a continuous current passing in the same direction as the discontinuous one. It is a curious fact, that this augmentation remains some instants after the continuous current has ceased to pass ; and that it disappears, not all at once, but by degrees and by fits.

I performed these experiments with steel wires of $I$ and 2 millimetres in diameter, with rods, both tempered and not tempered, of 3 to 4 millimetres in diameter. The results were the same; the strengthening due to the continuous current is more decided with the rods than with the wirest.

* This experiment of the iron wire covered with silk its whole length, and which gives all the same sounds as when its surface is perfectly naked, would seem to indicate that external causes, such as friction, enter less than is imagined into the production of these sounds : this point deserves to be more closely examined.

+ The steel rods are magnetized by the simple passage of these disconti- 
It seems to me to result from what precedes, that the effect of the transmitted current is to give to the molecules of the iron a transversal direction, as the effect of the magnetization was to give them a longitudinal one. If the arrangement of the particles of the iron-filings around an iron or any other metallic wire, traversed by an electric current, be examined with attention, these filings are seen to arrange themselves in parallel transversal lines. This is very evident on fixing the conducting wire into a groove made in a wooden board. The filings, being unable to go round the wire, assume a transverse position above it, forming small threads of 3 to 4 millimetres in length, which present opposite poles at their two extremities. When the wire is free, these threads, instead of remaining rectilinear, unite at their two ends, and envelope the surface of the wire, forming around it a closed curve. Now the arrangement which the iron-filings assume around any conducting wire transmitting a current, should be equally assumed by the molecules of the surface of a soft iron wire itself traversed by a current, owing to the effect of this current transmitted through the whole mass of the wire. This is moreover proved by the experiments of Mr. Joule, which show that a soft iron wire or rod experiences a shortening from the effect of a transmitted current. It results, therefore, that when the transmitted current is intermittent, the particles of the surface oscillate between that transversal position and their natural one, and that consequently there is a production of vibrations. These oscillations are the more easy, and consequently the vibrations are the stronger, in proportion to the softness of the iron; with hardened iron, and especially with hard steel, there is a greater resistance to overcome, and the effect is consequently less perceptible. In the first case, the transmission of a continuous current, by impressing on the particles in a permanent manner the position which the discontinuous current tends to give them, must annul or at least materially diminish the oscillatory movement; this in fact takes place. In the case of the hardened iron or steel, the continuous current must, on the contrary, by disturbing the particles from the normal position, without however being able completely to impress on them the transversal direction, on account of the too great resistance which they offer to a displacement, facilitate the oscillatory action of the discontinuous

nuous currents without external current; but they acquire numerous poles. Is this magnetization attributable to the effect of the terrestrial magnetism which the molecular vibrations experienced by the steel would favour, or is it a direct effect of the current transmitted intermittently? New researches would be necessary to solve this question. 
current; experiment confirms this. With regard to the in. terruptions in the intensity of the sound in this last case, after the continuous current has ceased to pass, they are probably attributable to the fact that the particles, disturbed from their natural position for a longer or shorter time, only return after a more or less prolonged series of oscillations, which the uninterrunted action of the discontinuous current favours.

In aid of the explanation which $I$ have just given, I will add that, having covered a copper wire with an iron envelope which was contiguous to it, and, so to say, plated, I obtained, by passing the discontinuous current through the copper wire, the same effects, excepting intensity, as if the wire had been entirely of iron; only the sound was not musical, but resembled that which would have been emitted by filings strongly agitated. As this result might be attributed to the fact that a part of the current traversed the iron covering itself, instead of circulating exclusively through the copper wire, I isolated this last by means of a layer of silk or wax, so that the thin cylinder of sheet-iron which surrounded it was not in metallic communication with the copper. The effect was exactly the same as in the preceding case; that is to say, the copper wire being traversed by a discontinuous current, caused a series of vibrations, or dry and metallic sounds, in the iron covering. This covering underwent, therefore, a transversal magnetization analogous to that which the surface of a wire entirely of iron experiences; this, indeed, was easily proved from the fact, that the iron-filings were attracted on the two sides of a small longitudinal slit which this covering presented in some parts of its surface, and that the two margins possessed an opposite magnetic polarity. All these effects were more marked when a continuous current, going in the same direction as the discontinuous one, was transmitted through the copper wire; they ceased entirely as soon as the two currents no longer passed. This increase in intensity of the sound produced by the passage of the continuous current was due to the fact that the discontinuous current had not alone, from acting at a distance, power enough to surmount the coercitive force of the iron covering, which itself was very much hardened, and that it needed the aid of the continuous current to impress a transversal position on the particles of the iron.

Before concluding this first part of my researches, I must again observe, that the passage of a continuous current, passing in a contrary direction to the discontinuous one, diminishes the sound instead of destroying it completely, when the wire submitted to the experiment is of soft iron; it modifies without 
perceptibly weakening it in the steel wires and rods; lastly, it causes it completely to disappear in the iron tube through which the conducting copper wire covered with silk passes. These effects vary, moreover, with the absolute intensity of the currents employed; and they are easily interpreted on the principles which we have stated. We must, moreover, not lose sight of the fact, that when the continuous and discontinuous currents are of equal force, they destroy one another whenever they pass together; so that the discontinuous one no longer acts, and the continuous one becomes intermittent in its action.

Lastly, permanent magnetization materially modifies the sound which the passage of the discontinuous current causes a soft iron wire or rod to emit. In order to guard, in this experiment, against the mechanical effects due to magnetization, I placed a soft iron rod on the two poles of an electromagnet, taking care by means of an interposed sheet of paper to avoid metallic contact between the poles and the rod. I then placed upon it considerable weights, that its position might not be modified by the magnetization, and passed the discontinuous current through the rod; it gave out a succession of dry metallic sounds, which became much more intense and grave at the moment when I magnetized the electro-magnet. It is evident that this modification and this strengthening of the sound are caused by the contest which is established between the longitudinal position which the influence of the magnetization impresses on the particles of the soft iron, and the transversal position which the passage of the current tends to give them; the oscillations of the particles must necessarily have more amplitude, since they take place between these extreme portions. The effect of permanent magnetization, although still perceptible, is less marked with steel rods, and especially with those of tempered steel.

\section{\$ II. Investigation of the vibratory movements which non-mag- netic bodies experience under the influence of external and transmitted electric currents.}

I stated in a preceding memoir, that rods, even of a tolerable size, of different non-magnetic metals emitted a distinct sound when, after having placed them under and very near an electromagnet or in the interior of the axis of a helix, a discontinuous current was made to pass through them. The sound only became perceptible, whatever was the force of the transmitted current, at the instant when the wire of the electro-magnet or that of the helix was traversed by a continuous current. I also observed that the effect was still more marked when the metal was 
in the form of a tube, or of a large wire turned into a helix. I likewise convinced myself, by employing rods of large dimensions, that the production of the sound could not be attributed either to a calorific effect of the current, nor to a mechanical action exerted by the electro-magnet or by the helix upon the conductors traversed by the discontinuous current. I have since made some new observations which sufficiently show that this kind of action is of a particular nature, probably molecular, like that which takes place in magnetic bodies.

Let us first remark, that with a single discontinuous current the phænomenon may be produced without having need in addition of a continuous current or of an electro-magnet. It suffices, for this, to twist the wire which conducts the discontinuous current into the form of a helix. A magnet is thus created; for every time that the current traverses the helix, the latter acquires magnetic properties, and at the same time the wire of the helix is itself a conductor traversed by the discontinuous current upon which the whole of the helix considered as a magnet acts. Moreover, every helix constructed of any metallic wire, the coils of which, whether covered or not with silk, are more or less pressed together, emits a very distinct sound when it is traversed by a discontinuous current. A continuous current, transmitted in the same direction as the discontinuous one, causes the sound to cease entirely, or diminishes its intensity materially, although a notable increase results from it in the electro-magnetic intensity of the helix. This neutralizing effect is probably owing to the fact, that the continuous current impressing permanently on the particles of the wire the position which they should take under the magnetic influence which the helix itself exerts, the discontinuous current no longer causes, at the instant when it is transmitted, new displacements. This effect is perfectly similar to that which is exerted by a continuous current when it passes through an iron wire traversed by a discontinuous current; it causes a cessation of the sound which this wire made under the action of the last current.

But if the continuous current, instead of traversing the wire of the same helix which conducts the discontinuous current, circulates through the wire of another belix which surrounds the first, the effect is totally different. In this case the sound is perceptibly increased, and becomes in general more metallic; this increase is more perceptible in proportion to the weakness of the discontinuous current, and the energy of the ambient continuous current. In this experiment the two currents pass in the same direction; so that the two helices, considered as magnets, present each the same magnetic pole at their same 
extremities. If the ambient continuous current proceeds in a contrary direction to the discontinuous one, the sound is weaker than in the preceding case, but stronger than when the exterior helix does not act; it produces likewise a different impression: it resembles the noise of water on the boil, whereas before it seemed to resemble that which a succession of strong sparks would have occasioned. We must remark, however, that if the discontinuous current is powerful enough to cause of itself a sufficiently intense sound to be easily heard at some feet distant, the influence of an external continuous current, when it passes in a contrary direction, diminishes the intensity of the sound. It always increases it, and in all cases, when the two currents pass in the same direction.

The continuous current may also be transmitted through the internal helix, and the discontinuous one through the external helix, contrary to what took place in the preceding experiments. In this case, if the two currents pass in the same direction, the sound resembles a succession of shocks; and when they pass in a contrary direction, the noise is only slightly increased, unless the discontinuous current is produced by a weak battery, for example, by a couple of Grove's cells: then the increase takes place also; only the noise resembles that of water on the boil.

It is easy to understand how a helix traversed by a continuous current, and placed externally or internally to the helix traversed by the discontinuous current, increases the sound which this latter emits. In fact, a permanent magnet is in this case created, the action of which is added to that of the temporary magnet which the discontinuous current creates when it passes through the helix. Moreover, in passing the continuous current through the wire of another helix than that which transmits the discontinuous one, the molecules of the conducting wire do not at first receive the position which they take only by the effect of the passage of the discontinuous current; this it is that causes them to oscillate freely around their natural position of equilibrium. The same result could not be obtained when the two currents passed through the wire of the same helix, as we have already remarked.

When the currents pass in contrary directions in the two helices, it is evident that the result is a diminution in the total magnetism of the whole of the two helices placed in juxtaposition: this is the reason that the sound is not generally increased; and it is only so when the discontinuous current being very weak, the magnetism of the helix which it traverses is so likewise. The magnetism of the other helix, traversed 
by the continuous current more energetic than the discontinuous one, then exerts a preponderating influence.

With respect to the difference in the nature of the sound, according as the continuous current passes in the same direction as the discontinuous one, or in a contrary direction, I can only explain it by admitting that the influences of the two magnetisms, opposed and unequal in intensity; diminish the amplitude of the oscillations which the particles of the wire traversed by the discontinuous current make around their natural position of equilibrium, - an amplitude which, on the contrary, is more considerable when the two magnetisms act in the same direction.

I placed a tube of soft iron between the two helices; an increase in the intensity of the sound took place when the two currents proceeded in the same direction,- a diminution and even a complete annihilation when they went in a contrary direction. If the soft iron tube is longer than the internal helix, its presence completely intercepts the influence of the external helix traversed by the continuous current: this is not the case if the tube is slit lengthwise ; its presence then in no degree modifies the phænomena. On placing the tube, or a massive cylinder of soft iron in the axis of the internal helix, and no longer between the two helices, the sound is perceptibly increased, especially when the continuous current traverses the internal helix, and the discontinuous one the external helix; the increase takes place even when there is only a discontinuous current. In all cases the nature of the sound does not change; its intensity alone is modified.

A tube of copper or of any other metal produces no effect when placed in the axis of the internal helix. This is not the case if it is between the two helices; it then causes a new sound: this sound is evidently attributable to a current of induction which circulates around the copper tube; for if this tube is slit in its whole length, there is no longer any sound, even when, to increase the magnetic force of the helix, a cylinder of soft iron is placed in its axis.

It is not necessary that the conductors traversed by a dis. continuous current should have the form of wires or that of a rod, to give out a sound under the influence of a magnet or a continuous current. A brass disc, 12 centimetres in diameter and 1 millimetre in thickness, was placed horizontally in the centre of a helix, by means of a vertical metallic support which was fixed in the centre of the disc. Care was taken to avoid any contact between the sides of the disc and the internal surface of the helix; the discontinuous current was directed from the centre to the circumference, or from the 
circumference to the centre. As soon as a continuous current was transmitted through the wire of the ambient helix, a wellmarked succession of metallic sounds was heard in the disc.

Even mercury can produce sounds, as I have already remarked. To render them perceptible, it is only requisite to introduce the mercury into a tube a few millimetres in diameter, and shaped so as nearly to envelope the pole of the electromagnet. As soon as the latter is magnetized, and the discontinuous current traverses the mercury, a series of sounds is heard similar to those which would result from a regular succession of sparks produced by a strong current between the mercury and a metallic wire. There is not, however, any trace of this, as may be easily ascertained; and, moreover, a current too feeble to produce sparks passed through the mercury is capable of producing the phænomenon. A remarkable fact is, that if, instead of being a little below the polar surface of the electro-magnet, the tube which contains the mercury is upon that surface itself, the sound is not heard. All these effects are very distinct from the movement which the mercury acquires under the influence of magnets, when it is traversed by either discontinuous or continuous currents.

I shall not for the present recur to the remarkable sounds which the voltaic arc occasions under the influence of the electro-magnet,-sounds which $I$ have carefully described in a previous memoir*. They are evidently of the same nature as those of which I have just spoken; for in the voltaic arc the current is, so to say, intermittent from the very nature of the arc which conducts it.

The following, therefore, is the general phænomenon. When any solid conductor, liquid or gaseous (at least very much divided, as in the voltaic arc), is traversed by an electric current, - a magnet, or an assemblage of electric currents closed, and having consequently magnetic properties, acts upon the particles of this conductor so as to give them a relative position different from that which they have naturally. Hence it results that, if the transmitted current is discontinuous, the particles oscillate between their normal position and the forced position which the magnetic influence tends to impart to them ; this gives rise to the sounds, and explains the modifications which it presents.

Does the action of the magnetism alone suffice to alter the relative position of the particles of all the bodies, or is the combined action of the magnetism and the electric currents requisite? Faraday's experiments seem to favour the first hypothesis; for the action exerted on light by transparent

* Phil. Mag. vol. xxxi. p. 321. 
bodies subjected to the influence of a strong electro-magnet clearly indicate a molecular derangement in them. The recent researches of $M$. Matteucci, showing that a mechanical action, such as compression, may annul or increase on the same substance (heavy glass, for example) the effect obtained by Faraday by means of an electro-magnet, confirm the fact that this last effect is also a molecular phænomenon.

With regard to non-transparent but conducting bodies, if they are magnetic, they experience a molecular modification under the action of the magnet, as we have stated above. If they are not magretic, they are dianagnetic; and then it is very probable that the diamagnetism causes a change in the position of their particles, as magnetism does in the others; for it appears to be satisfactorily proved that diamagnetism is attributable to a transversal polarity, as magnetism is due to a longitudinal polarity*. Now whenever any cause produces a polarity in the particles, these must, in obedience to it, arrange themselves in a determined relative position exactly as takes place in the phænomenon of crystallization.

It is therefore probable that, under the influence of a magnet or of an external current, the particles of a diamagnetic body tend to arrange themselves transversely; whilst under that of a transmitted current, they take a longitudinal direction, as is shown by the force of projection which carries the particles of a conductor from the positive to the negative pole, at the point where the circuit is interrupted, in giving rise to the voltaic arc. The struggle between these two contrary tendencies, the one transversal, the other longitudinal, produces the uscillatory movements of the particles around their position of equilibrium, and consequently the vibrations.

In the production of the currents of induction, this molecular derangement which is produced by the magnetic action of a magnet or of a closed current must necessarily take place. To this derangement is probably due the production of an instantaneous electric current; just as when the molecular derangement ceases on the disappearance of the cause which has determined it, there arises a second electric current, having a contrary direction to the first. It is easy to understand why these two currents pass thus in opposite directions.

A conductor placed under the influence of an electromagnet, or of molecular currents, must therefore be, as long as that influence is exerted upon it, in peculiar molecular conditions. This is shown by the affections of light in those which are transparent; it remains to be demonstrated by other

* The recent researches of Prof. Faraday on the magnetic polarity of crystals of bismuth are quite favourable to this view.

Phil. Mag. S. 3. Vol. 35. No. 238. Dec. 189. 
direct means, as to those which are opake, as Mr. Joule has done for magnetic substances. For the proofs drawn from diamagnetism and sonorous vibrations are only indirect, although the last appear to be tolerably conclusive.

I shall soon return to this subject, in reference to some researches on the relation between diamagnetism and the induced currents, on which I am at present engaged.

LII. On Systems of Algebra involving more than one Imaginary; and on Equations of the Fifth Degree. By JAMES Cockle, Esq., M.A., Barrister-at-Law*.

CONCEIVE two imaginaries, such that their respective squares are equal either to positive or to negative unity. Then the product of two linear functions of these imaginaries is not of the same form as its factors. The product of the imaginaries prevents this similarity, and obstructs the formation of a System of Triple Algebra on the basis just mentioned. But, if we invest the last-named product with the character of a third imaginary, and assume that its square is equal either to positive or to negative unity, four systems of Quadruple Algebra will present themselves, in each of which the product of two linear functions of the three imaginaries will, in general, have the same form as its factors.

Let $\alpha$ and $\beta$ respectively represent the first and second, and $\gamma$ the third imaginary. Then $\gamma=\alpha \beta$ or $\beta \alpha$. But as, in quadruple algebra, $\alpha \beta$ is not always equal to $\beta \alpha$, I shall select the former as the expression for $\gamma$.

Let $A$ denote a linear function of $\alpha, \beta$, and $\gamma$; in other language, let

$$
A=w+\alpha x+\beta y+\gamma z,
$$

then, in one of the four systems of quadruple algebra above alluded to, the expression $A$ bears the name of a quaternion. In the remaining three systems the respective terms tessarine, coquaternion, and cotessarine may be applied to it. At least I have suggested such a nomenclature in No. 1360 of the Mechanics Magazinet, where I have shown the existence of

* Communicated by the Author. In connexion with his paper published at pp. 406-410 of the preceding (34th) volume of this Journal, Mr. Cockle is desirous of referring the reader to two articles subsequently comnunicated by him to the Mechanics' Magazine, and which will be found at pp. 534 and 558,559 of vol. 50 of that work.

+ See pp. 197,198 of the current (5lst) volume of that work. I had, however, previously employed the term "Tessarine" both in that and in the present Journal. 